\title{
Long-term outcome of adrenalectomy for metastasis resulting from colorectal cancer with other metastatic sites: A report of 3 cases
}

\author{
MAMORU UEMURA ${ }^{1,2^{*}}$, HO MIN KIM ${ }^{3 *}$, MASATAKA IKEDA ${ }^{2}$, JUNICHI NISHIMURA $^{1}$, TAISHI HATA ${ }^{1}$, \\ ICHIRO TAKEMASA ${ }^{1}$, TSUNEKAZU MIZUSHIMA ${ }^{1}$, HIROFUMI YAMAMOTO ${ }^{1}$, \\ YUICHIRO DOKI $^{1}$ and MASAKI MORI ${ }^{1}$ \\ ${ }^{1}$ Department of Gastroenterological Surgery, Graduate School of Medicine, Osaka University, Suita, Osaka 565-0871; \\ ${ }^{2}$ Department of Surgery, Osaka National Hospital, Osaka, Osaka 540-0006; \\ ${ }^{3}$ Department of Surgery, Rinku General Medical Center, Izumisano, Osaka 598-8577, Japan
}

Received February 18, 2015; Accepted January 26, 2016

DOI: $10.3892 / \mathrm{ol} .2016 .4897$

\begin{abstract}
Metastasis to the adrenal glands is a relatively frequent observation at autopsy of patients that have succumbed to cancer. Long-term disease-free survival has been reported in patients following the resection of solitary adrenal metastasis resulting from colorectal cancer. In addition, following primary resection for colorectal cancer, solitary metastasis to the adrenal glands is rare, even in outpatients at routine follow-ups. Therefore, adrenal metastasis is usually detected in combination with multiple synchronous metastases at other sites in the terminal stages of cancer. Between 1998 and 2002, 3 patients with adrenal metastasis and other synchronous metastatic sites underwent surgery for adrenal metastasis at the Department of Gastroenterological Surgery at Osaka University. The other synchronous metastatic sites observed in the 3 patients consisted of lung and para-aortic lymph nodes. In total, 2 out of the 3 patients experienced long-term disease-free survival for $>5$ years following surgery and 1 patient underwent curative resection for recurrence of metastases in the liver and right adrenal gland 79 months subsequent to the initial resection for adrenal metastasis. All 3 patients survived for $>90$ months. In conclusion, aggressive surgical resection for adrenal metastasis and other metastatic sites resulting from colorectal cancer may result in a survival benefit in selected patients.
\end{abstract}

Correspondence to: Dr Masataka Ikeda, Department of Surgery, Osaka National Hospital, 2-1-14 Hoenzaka, Osaka, Osaka 540-0006, Japan

E-mail: mikeda@onh.go.jp

*Contributed equally

Key words: colorectal cancer, adrenal metastasis, long-term survival

\section{Introduction}

Cancer is a major public health issue in developed countries (1). In Japan, the incidence of colorectal cancer (CRC) has significantly increased in recent years (2). The most critical factor causing mortality of patients with CRC is metastasis (3). Intensive follow-up and adjuvant therapy is urgently required for patients that undergo curative resection for $\mathrm{CRC}$, in order to prevent recurrence and metastasis (4-6). According to previous studies, CRC metastasis to distant organs, including the liver and lung, is resectable (7-11), and a successful resection clearly results in an increased survival time. Long-term survival has been reported in association with surgical resection for solitary adrenal metastasis resulting from CRC (12-22). However, isolated adrenal metastasis is rare; adrenal lesions usually occur in the presence of multiple synchronous metastases and are detected in the terminal phase of cancer (23-25). Therefore, surgical resection is not generally considered as a treatment for adrenal metastasis. However, several studies have reported that there is a favorable prognosis and notable benefit to patients following surgical resection for adrenal metastasis. To associate the features of adrenal metastasis and the clinical outcome of patients with adrenal metastasis resulting from $\mathrm{CRC}$, the present study reports the cases of $3 \mathrm{CRC}$ patients that experienced a long-term survival following surgical resection for adrenal metastasis with local and other metastatic sites, including the liver, lung and distant lymph nodes.

\section{Case report}

Between 1998 and 2002, 3 patients underwent surgery for adrenal metastasis at the Department of Gastroenterological Surgery at Osaka University (Suita, Osaka, Japan). All resected specimens were diagnosed according to the tumor-node-metastasis (TNM) classification $(26,27)$. Following surgery, the patients were followed-up using serological examinations, including serum carcinoembryonic antigen (CEA) and cancer antigen 125, and imaging modalities, such as abdominal ultrasonography, computed tomography (CT) and chest X-ray, 
every 3-6 months. The therapies the patients received were administered according to the Japanese guidelines for CRC treatment (7). Table I summarizes the clinical data of the 3 patients, who currently remain within the standard follow-up period following curative surgery (5 years).

Case 1. In May 2000, a 63-year-old man presented with locally recurrent rectal cancer subsequent to an anterior peritoneal resection conducted in July 1998. According to the TNM classification $(26,27)$, the pathological staging of the tumor in was stage IIIa [well-differentiated adenocarcinoma; pT3pN1M0; residual tumor (R) 0]. In May 2000, the CEA level of the patient had increased to $16 \mathrm{ng} / \mathrm{ml}$ (normal range, 0.0-4.0 ng/ml). An abdominal CT scan (Discovery CT750 HD; GE Healthcare, Piscataway, NJ, USA) identified the presence of local recurrence, which was posterior to the bladder. The patient refused treatment. The patient presented to hospital 1 year later with symptoms of anal pain and hematuria. The CEA level of the patient had increased to $110 \mathrm{ng} / \mathrm{ml}$ and the previously observed local recurrence had increased in size to a $60-\mathrm{mm}$ diameter from an original size of $20-\mathrm{mm}$ diameter (Fig. 1A). Beginning in July 2001, 40 Gy of external radiation in 20 fractions and 30 Gy of interstitial radiation therapy in 5 fractions was administered to the recurrent tumor. The CEA level of the patient $(8 \mathrm{ng} / \mathrm{ml})$ had decreased by October 2001. However, in August 2002, the CEA level of the patient increased to $11 \mathrm{ng} / \mathrm{ml}$ and an abdominal CT scan revealed left adrenal metastasis (Fig. 1B). The patient underwent laparoscopic left adrenalectomy. A pathological examination of the tumor demonstrated a well-differentiated adenocarcinoma consistent with primary CRC. The patient was followed up without adjuvant chemotherapy following adrenalectomy, and was alive and well at the last follow-up in April 2012, with no evidence of recurrence observed using positron emission tomography (PET)-CT (HEADTOME/set. 2400W; Shimadzu Co., Kyoto, Japan) and serum CEA, 9.5 years subsequent to the resection of the adrenal metastasis (Fig. 1C).

Case 2. A 62-year-old man underwent low anterior resection for rectal cancer in July 2000. According to the TNM classification $(26,27)$, the post-operative staging of the tumor was stage II (well-differentiated adenocarcinoma; pT3pNOM0; R0). Subsequent to 5 months, abdominal and chest CT (Discovery CT750 HD; GE Healthcare) revealed the presence of a 30-mm mass near the anastomotic region in the abdominal cavity and a $10-\mathrm{mm}$ mass in the lung, consistent with pulmonary metastasis. A total of 4 cycles of chemotherapy [ $750 \mathrm{mg} 5$-fluorouracil (5-FU) with $400 \mathrm{mg}$ leucovorin (LV)] were administered and the pulmonary metastasis decreased in size. However, the local recurrence increased to $50 \mathrm{~mm}$ in diameter (Fig. 2A). Therefore, $50 \mathrm{~Gy}$ of external radiation in 25 fractions was initiated, which resulted in no alteration in the local recurrence. Additional chemotherapy [400 mg tegaful/uracil (UFT) with $180 \mathrm{mg}$ camptothecin-11 (CPT-11)] was administered for 5 cycles; subsequently, the local recurrence appeared as scar tissue on CT scans and the serum CEA level of the patient gradually decreased from 19.1 to $1.3 \mathrm{ng} / \mathrm{ml}$, which was within the normal range ( $\leq 3.0 \mathrm{ng}$ / $\mathrm{ml})$. Abdominal and chest $\mathrm{CT}$ were performed 10 months later and a solitary pulmonary metastasis $18 \mathrm{~mm}$ in size was revealed in the right lower lobe and the presence of left adrenal metastasis

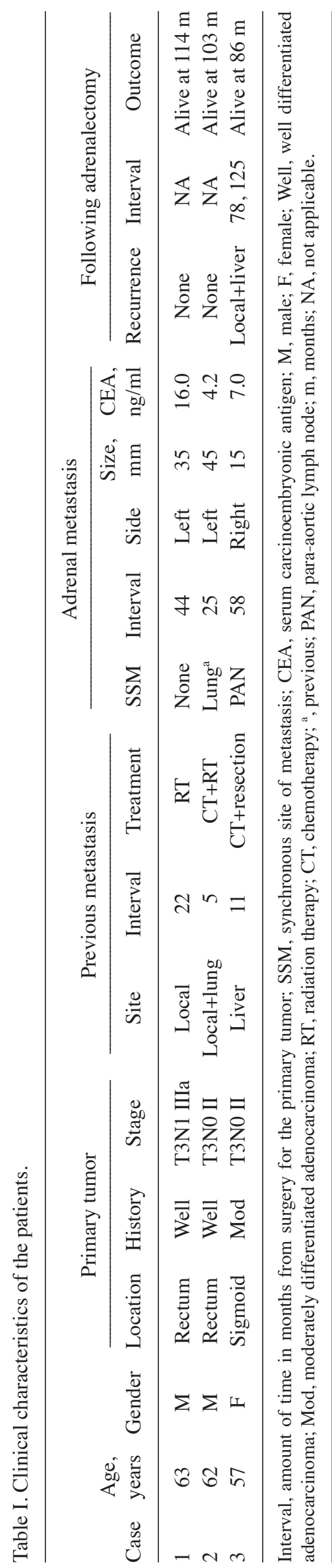


A
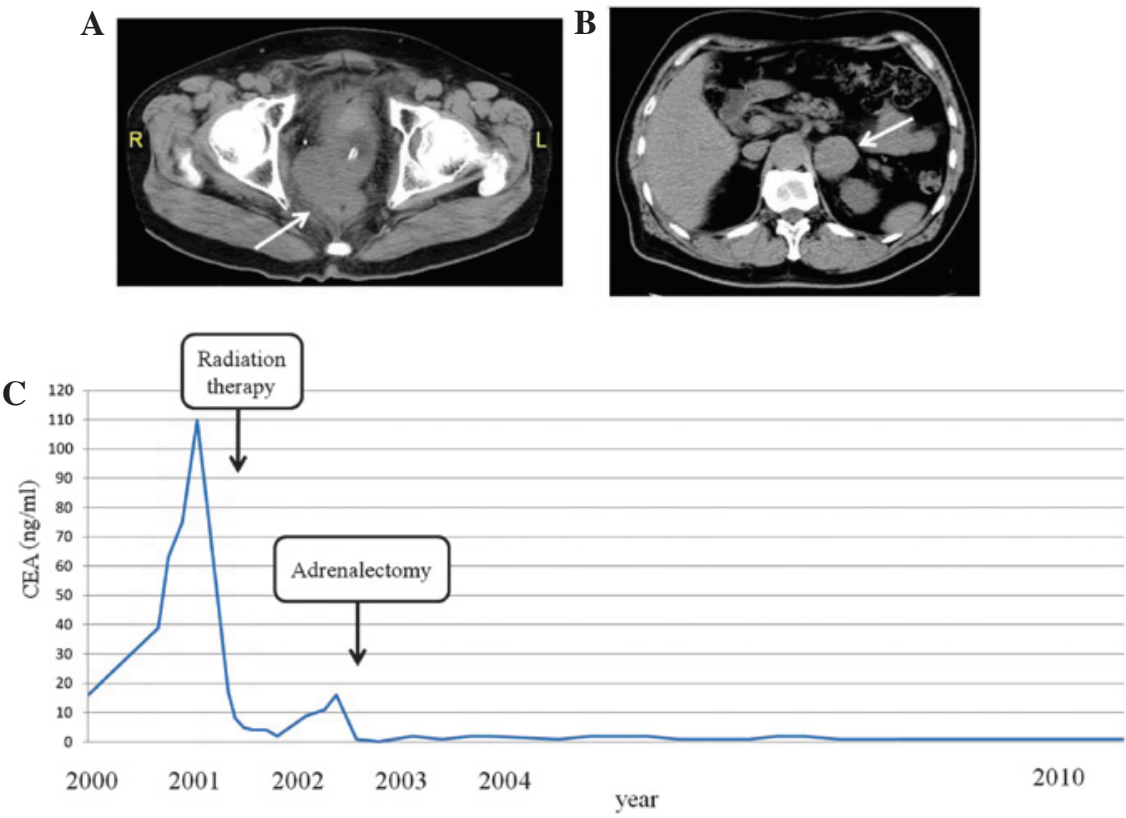

Figure 1. Patient 1. (A) Abdominal CT of local recurrence behind the bladder, indicated by an arrow. (B) Abdominal CT of an enlarged left adrenal gland, indicated by an arrow. (C) Alterations in the serum CEA levels of the patient. CT, computed tomography; CEA, serum carcinoembryonic antigen.
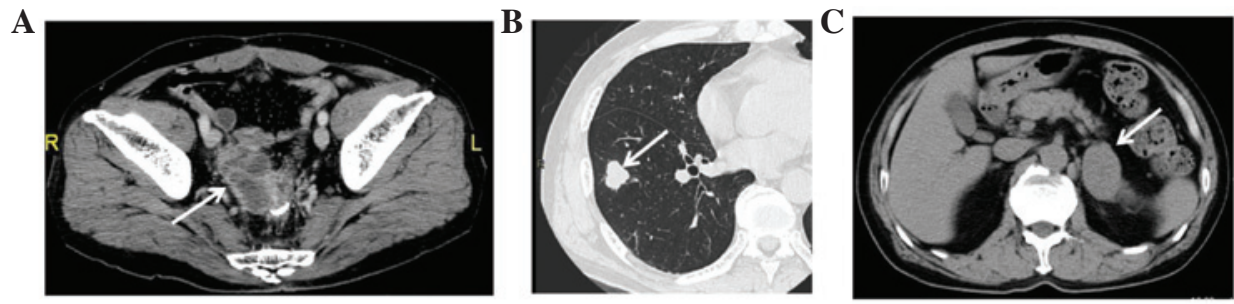

D

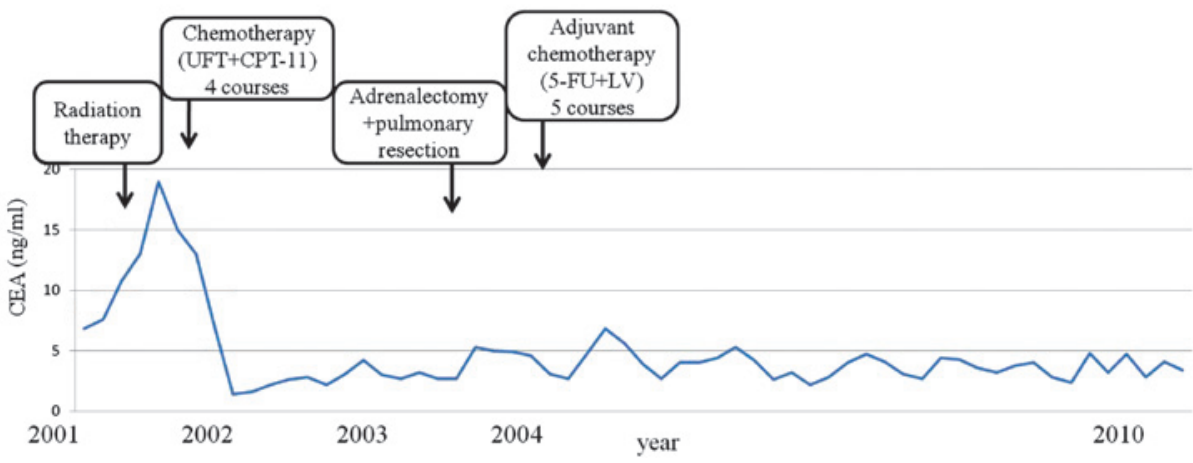

Figure 2. Patient 2. (A) Abdominal CT of local recurrence near the anastomotic lesion, indicated by an arrow. (B) Chest CT of the right lung nodule, which was $18 \mathrm{~mm}$ in diameter, indicated by an arrow. (C) Abdominal CT of an enlarged left adrenal gland, indicated by an arrow. (D) Alterations in the serum CEA levels of the patient. CT, computed tomography; CEA, serum carcinoembryonic antigen; UFT, tegaful/uracil; CPT-11, camptothecin-11; F-FU, 5-fluorouracil; LV, leucovorin.

(Fig. 2B and C). Left adrenalectomy and partial right lower lobe pneumonectomy were performed in September 2003. A pathological examination confirmed that the adrenal gland and pulmonary nodules were well-differentiated adenocarcinoma consistent with metastatic CRC. A total of 3 cycles of 5-FU with LV were administered as adjuvant chemotherapy following the second surgery. At the last follow-up in May 2012, the patient was alive and without evidence of metastasis observed using PET-CT (HEADTOME/set. 2400W; Shimadzu Co.) and with a normal CEA level for almost 8.6 years following the second surgical resection of adrenal and pulmonary metastases (Fig. 2D).
Case 3. A 57-year-old woman underwent a sigmoidectomy in January 2002 for CRC. According to the TNM classification $(26,27)$, the pathological staging of the tumor was II (moderately-differentiated adenocarcinoma; pT3pNOM0; R0). Eleven months after sigmoidectomy, abdominal CT (Discovery CT750 HD; GE Healthcare) revealed liver metastasis [segment (S) 2 and S7], and 4 cycles of chemotherapy (400 mg UFT with $240 \mathrm{mg}$ CPT-11) were administered to the patient for 5 months. After 4 cycles of chemotherapy, the liver metastasis decreased in size and new lesions were not detected. A partial liver resection (S2 and S7) was performed 

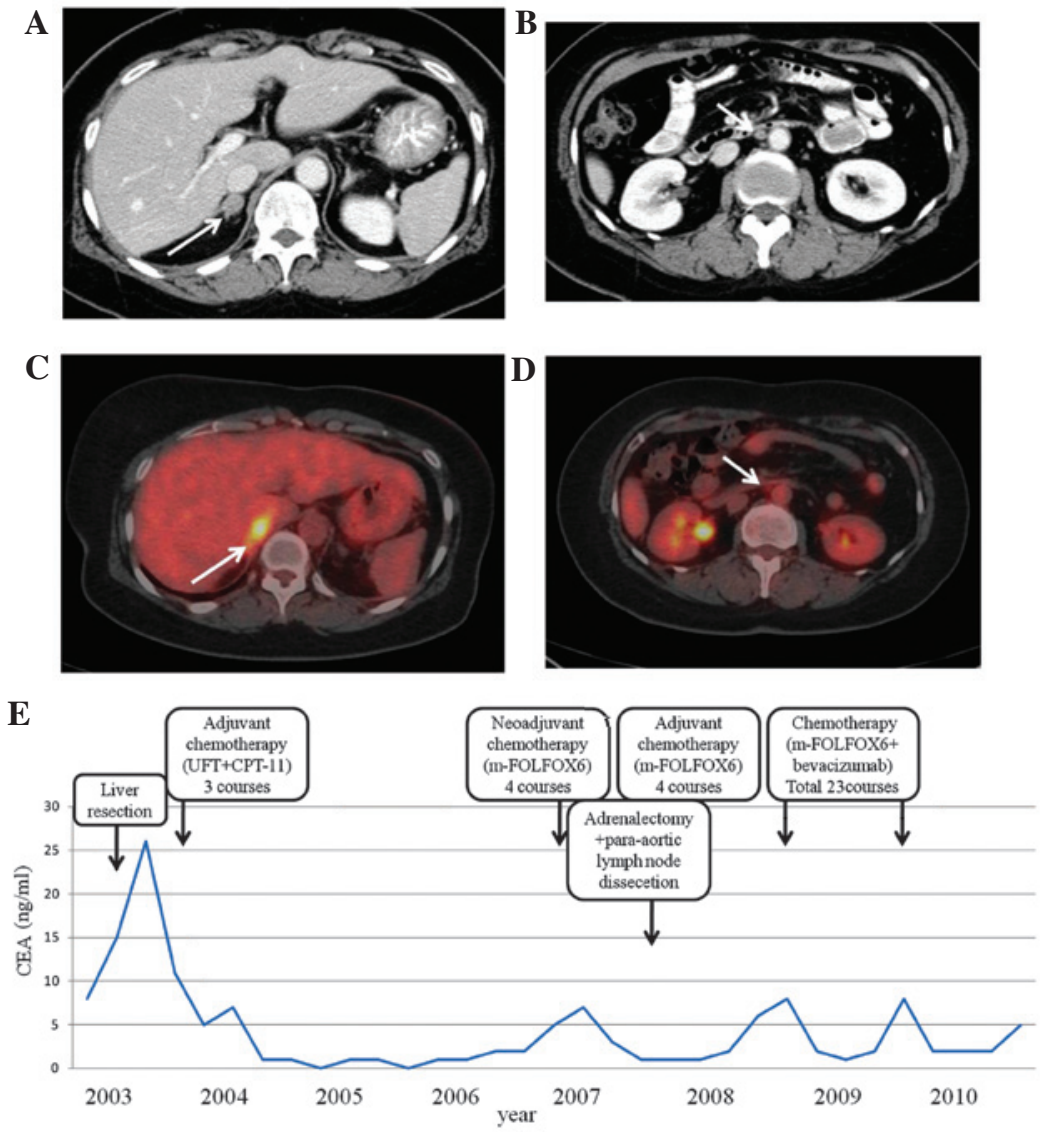

Figure 3. Patient 3. (A) Abdominal CT of an enlarged right adrenal gland, indicated by an arrow. (B) Abdominal CT of para-aortic lymph node swelling, indicated by an arrow. (C) Abdominal PET-CT with FDG uptake of right adrenal gland, indicated by an arrow. (D) Abdominal PET-CT of the para-aortic lymph node with FDG uptake, indicated by an arrow. (E) Alterations in the serum CEA levels of the patient. CT, computed tomography; PET, positron emission tomography; FDG, [18F]-2-fluoro-2-deoxy-D-glucose; CEA, serum carcinoembryonic antigen; UFT, tegaful/uracil; CPT-11, camptothecin-11; mFOLFOX6, modified FOLFOX6 regimen (5-fluorouracil with leucovorin and oxaliplatin).

in July 2003, and 3 additional cycles of chemotherapy (400 mg UFT with $180 \mathrm{mg}$ CPT-11) were administered. Forty months subsequent to the second surgery, PET-CT (HEADTOME/ set. $2400 \mathrm{~W}$; Shimadzu Co.) revealed the presence of adrenal metastasis and para-aortic lymph node recurrences (Fig. 3A-D). In total, 4 cycles of the modified folinic acid, 5-FU and oxaliplatin (mFOLFOX6) regimen (5-FU with LV and oxaliplatin) were administered to the patient as pre-operative chemotherapy. In each course, there were no cycles, but simply one rapid infusion and one continuous infusion for $46 \mathrm{~h}$. The adrenal metastasis and lymph node recurrence decreased in size, and a right adrenalectomy and para-aortic lymph node dissection was performed in July 2007. A pathological examination of the adrenal gland confirmed the diagnosis of moderately-differentiated adenocarcinoma consistent with metastatic CRC. Metastasis was detected in 1 out of 13 dissected para-aortic lymph nodes. mFOLFOX6 was administered as post-operative chemotherapy for 4 cycles following the third surgery. Subsequently, the CEA level of the patient was elevated to $8 \mathrm{ng} / \mathrm{ml}$ and PET-CT revealed a local recurrence in the right adrenal gland. Therefore, mFOLFOX6 with bevacizumab was administered to the patient for 23 cycles and discontinued following the development of adverse reactions, including peripheral neuropathy (grade 2), general fatigue and nausea. Following mFOLFOX and bevacizumab treatment for the local adrenal recurrence, the patient was well and no evidence of an additional recurrence was observed using PET-CT (Fig. 3E). However, 79 months subsequent to the initial resection of adrenal metastasis, the patient underwent a curative resection for recurrence of liver metastases and recurrence in the right adrenal gland. At the last follow-up in February 2015, the patient was alive and no evidence of metastasis was observed using PET-CT and the patient had a normal CEA level for 12 months following the second surgical resection of adrenal and liver metastases.

\section{Discussion}

According to the results from several studies, metastasis to the adrenal glands is a relatively frequent observation at autopsy in cancer patients (23-25). The most common neoplasms to metastasize to the adrenal glands are lung cancer, breast cancer and renal cell carcinoma (23-25). The incidence of adrenal metastasis observed at autopsy is $8.6-27.0 \%$ of all malignancies (23-25). The range of the incidence of adrenal metastasis resulting from CRC is $4.2-14.4 \%$ (23-25). Cedermark et al reviewed the autopsy records of 457 patients that succumbed to $\mathrm{CRC}$ and observed that the frequency of metastasis to the liver and lung was 48 and $38 \%$, respectively, whereas metastasis to the adrenal gland was $14 \%$ (25).

It is widely accepted that the serum CEA level is important in patients with $\mathrm{CRC}$, as it may affect tumor diagnostic 
procedures (28). In addition, the serum CEA level is reportedly useful for indicating the presence of adrenal metastasis following surgical resection for CRC $(13,19,20)$. In the present study, the post-operative recurrence was predicted from the alterations in the serum CEA level, and recurrence of the tumors was suspected when the CEA level became elevated. In patients 1 and 2, the serum CEA level was within normal limits for $>5$ years following the first surgery, and in patient 3 , recurrence was suspected following the third surgical resections when the CEA level increased. Therefore, a serial post-operative determination of the serum CEA level is a useful and effective guide for determining the status of patients with recurrent CRC.

All patients in the present study were asymptomatic, and did not present with abdominal pain or adrenal insufficiency, which has been reported in a previous study (22). It has been estimated that $80-90 \%$ of the adrenal gland must be replaced or destroyed by tumor cells prior to adrenal insufficiency being detected; therefore, symptoms rarely appear as an initial sign of adrenal metastasis resulting from CRC (22). The rate of detecting clinically silent adrenal masses has increased due to the widespread use of abdominal imaging modalities, including ultrasonography, CT, magnetic resonance imaging (MRI) and PET (29,30). Adrenal incidentaloma is defined as an adrenal mass detected during abdominal imaging performed for reasons not associated with the adrenal glands $(31,32)$. Certain adrenal incidentalomas are metastatic adrenal tumors from a different primary cancer, usually the lung or kidney. Among patients undergoing adrenalectomy for metastatic cancer, $13-17 \%$ of patients possessed metastasis resulting from CRC $(22,23)$. Candel et al conducted a study on fine-needle aspiration biopsies of adrenal masses in various malignancies, which revealed that 5 out of 39 cases (12.8\%) were derived from CRC (33). Frilling et al concluded that MRI and PET provided an accurate diagnosis in patients with indeterminate adrenal tumors (29). Even though the incidence of adrenal metastasis varies $(33,34)$, it is possible that adrenal metastasis from CRC is not unusual. Several studies identified that the majority of autopsy cases, where metastasis to the adrenal glands was observed, possessed several other metastases (23-25).

By contrast, a solitary adrenal metastasis is extremely unusual. When a solitary adrenal metastasis is observed, surgical resection of the involved adrenal glands is associated with a good prognosis for patients $(1,2,4-7,12-16,35-37)$. Muth $e t$ al reported that the factors associated with a longer survival time in patients with CRC, renal cell carcinoma, non-small-cell lung cancer and malignant melanoma were the tumor type, of which CRC demonstrated the best prognosis, no prior surgery or metastases, a long disease-free interval and potentially curative adrenalectomy at the time of surgery (32). Therefore, it is important to consider the possibility of adrenal metastasis resulting from CRC during follow-up subsequent to a primary surgery, since the early detection of a solitary adrenal metastasis may result in a second curative surgery and improve the long-term survival time of patients. Although adrenal metastasis is usually observed in combination with widespread metastasis $(23-25,38,39)$, it is considered feasible to resect a solitary adrenal metastasis in patients with CRC, which leads to an improved prognosis (12-22).
Recently, metastasis to the adrenal glands has been more frequently recognized during follow-up subsequent to surgery for cancer, due to an improvement in high-resolution imaging modalities (22). However, metastasis to the adrenal glands usually occurs in combination with multiple synchronous metastases at other sites $(13-26,38,39)$. In the present study, 2 out of 3 patients that possessed multiple metastases, underwent adrenalectomy for metastatic CRC and were alive without chemotherapy 8 years and 6 years subsequent to the last surgery, respectively. The third patient that underwent adrenalectomy was alive and undergoing mFOLFOX6 with bevacizumab treatment almost 3 years subsequent to the last surgery. The present results suggest that aggressive surgical resection for adrenal metastasis resulting from CRC may lead to a survival benefit in patients for whom curative treatment is performed at other metastatic sites. Surgical resection for metastasis to distant organs, including the liver and lung, from $\mathrm{CRC}$ is recommended, due to the numerous studies regarding these metastases (7-11). Similarly, adrenal metastasis resulting from CRC should be aggressively resected if distant metastasis may also be curatively treated.

In conclusion, aggressive surgical resection for adrenal metastasis resulting from CRC should be performed in certain patients, for whom curative treatment is performed at other metastatic sites.

\section{References}

1. Jemal A, Siegel R, Ward E, Hao Y, Xu J, Murray T and Thun MJ: Cancer statistics, 2008. CA Cancer J Clin 58: 71-96, 2008.

2. Kohno SI, Luo C, Nawa A, Fujimoto Y, Watanabe D, Goshima F, Tsurumi T and Nishiyama Y: Oncolytic virotherapy with an HSV amplicon vector expressing granulocyte-macrophage colony-stimulating factor using the replication-competent HSV type 1 mutant HF10 as a helper virus. Cancer Gene Ther 14: 918-926, 2007.

3. Steeg PS: Tumor metastasis: Mechanistic insights and clinical challenges. Nat Med 12: 895-904, 2006.

4. Wolpin BM and Mayer RJ: Systemic treatment of colorectal cancer. Gastroenterology 134: 1296-1310, 2008.

5. Kornmann M, Formentini A, Ette C, Henne-Bruns D, Kron M, Sander S, Baumann W, Kreuser ED, Staib L and Link KH: Prognostic factors influencing the survival of patients with colon cancer receiving adjuvant 5-FU treatment. Eur J Surg Oncol 34: 1316-1321, 2008.

6. Bathe OF, Dowden S, Sutherland F, Dixon E, Butts C, Bigam D, Walley B, Ruether D and Ernst S: Phase II study of neoadjuvant 5-FU + leucovorin + CPT-11 in patients with resectable liver metastases from colorectal adenocarcinoma. BMC Cancer 4: 32, 2004.

7. Watanabe T1, Itabashi M, Shimada Y, Tanaka S, Ito Y, Ajioka Y, Hamaguchi T, Hyodo I, Igarashi M, Ishida $\mathrm{H}$, et al; Japanese Society for Cancer of the Colon and Rectum: Japanese Society for Cancer of the Colon and Rectum (JSCCR) guidelines 2010 for the treatment of colorectal cancer. Int J Clin Oncol 17: 1-29, 2012.

8. Mori M, Tomoda H, Ishida T, Kido A, Shimono R, Matsushima T, Kuwano H and Sugimachi K: Surgical resection of pulmonary metastases from colorectal adenocarcinoma. Special reference to repeated pulmonary resections. Arch Surg 126: 1297-1302, 1991.

9. Regnard JF, Nicolosi M, Coggia M, Spaggiari L, Fourquier P, Levi JF and Levasseur P: Results of surgical treatment of lung metastases from colorectal cancers. Gastroenterol Clin Biol 19: 378-384, 1995 (In French).

10. van Ginkel RJ, de Jong KP, Peeters PM, de Vries EG and Slooff MJ: Good results with liver resection for colorectal liver metastases. Ned Tijdschr Geneeskd 139: 1546-1550, 1995 (In Dutch).

11. Ambiru S, Miyazaki M, Ito H, Nakagawa K, Shimizu H, Kato A, Nakamura S, Omoto H and Nakajima N: Resection of hepatic and pulmonary metastases in patients with colorectal carcinoma. Cancer 82: 274-278, 1998. 
12. Kanjo T, Albertini $\mathrm{M}$ and Weber S: Long-term disease-free survival after adrenalectomy for isolated colorectal metastases. Asian J Surg 29: 291-293, 2006.

13. Katayama A, Mafune K and Makuuchi M: Adrenalectomy for solitary adrenal metastasis from colorectal carcinoma. Jpn J Clin Oncol 30: 414-416, 2000.

14. Nagakura S, Shirai Y, Nomura T and Hatakeyama K: Long-term survival after resection of colonic adenocarcinoma with synchronous metastases to the liver, adrenal gland, and aortic-caval lymph nodes: Report of a case. Dis Colon Rectum 45: 1679-1680, 2002.

15. Mourra N, Hoeffel C, Duvillard P, Guettier C, Flejou JF and Tiret E: Adrenalectomy for clinically isolated metastasis from colorectal carcinoma: Report of eight cases. Dis Colon Rectum 51: 1846-1849, 2008.

16. Kosmidis C, Efthimiadis C, Anthimidis G, Levva S, Ioannidou G, Zaramboukas T, Emmanouilides C, Baka S, Kosmidou M, Basdanis G and Fachantidis E: Adrenalectomy for solitary adrenal metastasis from colorectal cancer: A case report. Cases J 1: 49, 2008.

17. Shoji Y, Dohke M, Masuda T, Nakamura F, Yano T, Niizeki H, Kashimura N and Matsunami O: Solitary adrenal metastasis in a patient with sigmoid colon cancer; report of a case. Int J Gastrointest Cancer 37: 120-123, 2006.

18. Murakami S, Terakado M, Hashimoto T, Tsuji Y, Okubo K and Hirayama R: Adrenal metastasis from rectal cancer: Report of a case. Surg Today 33: 126-130, 2003.

19. Watatani M, Ooshima M, Wada T, Terashita H, Matsuda T, Shindo K and Yasutomi M: Adrenal metastasis from carcinoma of the colon and rectum: A report of three cases. Surg Today 23: 444-448, 1993.

20. Fujita K, Kameyama S and Kawamura M: Surgically removed adrenal metastasis from cancer of the rectum. Report of a case. Dis Colon Rectum 31: 141-143, 1988.

21. Kim SH, Brennan MF, Russo P, Burt ME and Coit DG: The role of surgery in the treatment of clinically isolated adrenal metastasis. Cancer 82: 389-394, 1998.

22. Lam KY and Lo CY: Metastatic tumours of the adrenal glands: A 30-year experience in a teaching hospital. Clin Endocrino (Oxf) 56: 95-101, 2002

23. Abrams HL, Spiro R and Goldstein N: Metastasis in carcinoma; analysis of 1000 autopsied cases. Cancer 3: 74-85, 1950.

24. Bullock WKH and Hirst AE Jr: Metastatic carcinoma of the adrenal. Am J Med Sci 226: 521-524, 1953.

25. Cedermark BJ, Blumenson LE, Pickren JW, Holyoke DE and Elias EG: Ths significance of metastases to the adrenal glands in adenocarcinoma of the colon and rectum. Surg Gynecol Obstet 144: 537-546, 1977.
26. Eriksen MT, Wibe A, Norstein J, Haffner J and Wiig JN; Norwegian Rectal Cancer Group: Anastomotic leakage following routine mesorectal excision for rectal cancer in a national cohort of patients. Colorectal Dis 7: 51-57, 2005.

27. Ueno H, Mochizuki H, Hashiguchi Y, Ishiguro M, Miyoshi M, Kajiwara Y, Sato T, Shimazaki H and Hase K: Extramural cancer deposits without nodal structure in colorectal cancer: Optimal categorization for prognostic staging. Am J Clin Pathol 127: 287-294, 2007

28. Tan E, Gouvas N, Nicholls RJ, Ziprin P, Xynos E and Tekkis PP. Diagnostic precision of carcinoembryonic antigen in the detection of recurrence of colorectal cancer. Surg Oncol 18: 15-24, 2009.

29. Frilling A, Tecklenborg K, Weber F, Kühl H, Müller S, Stamatis G and Broelsch C: Importance of adrenal incidentaloma in patients with a history of malignancy. Surgery 136: 1289-1296, 2004.

30. Harrison J, Ali A, Bonomi P and Prinz R: The role of positron emission tomography in selecting patients with metastatic cancer for adrenalectomy. Am Surg 66: 432-436, discussion 436-437, 2000.

31. Wade TP, Longo WE, Virgo KS and Johnson FE: A comparison of adrenalectomy with other resections for metastatic cancers. Am J Surg 175: 183-186, 1998.

32. Muth A, Persson F, Jansson S, Johanson V, Ahlman H and Wängberg B: Prognostic factors for survival after surgery for adrenal metastasis. Eur J Surg Oncol 36: 699-704, 2010.

33. Candel AG, Gattuso P, Reyes CV, Prinz RA and Castelli MJ: Fine-needle aspiration biopsy of adrenal masses in patients with extraadrenal malignancy. Surgery 114: 1132-1136, discussion 1136-1137, 1993.

34. Sirén JE, Haapiainen RK, Huikuri KT and Sivula AH: Incidentalomas of the adrenal gland: 36 operated patients and review of literature. World J Surg 17: 634-639, 1993.

35. Strong VE, D'Angelica M, Tang L, Prete F, Gönen M, Coit D, Touijer KA, Fong Y and Brennan MF: Laparoscopic adrenalectomy for isolated adrenal metastasis. Ann Surg Oncol 14: 3392-3400, 2007.

36. Sebag F, Calzolari F, Harding J, Sierra M, Palazzo FF and Henry JF: Isolated adrenal metastasis: The role of laparoscopic surgery. World J Surg 30: 888-892, 2006.

37. Sarela AI, Murphy I, Coit DG and Conlon KC: Metastasis to the adrenal gland: The emerging role of laparoscopic surgery. Ann Surg Oncol 10: 1191-1196, 2003.

38. Lo CY, van Heerden JA, Soreide JA, Grant CS, Thompson GB, Lloyd RV and Harmsen WS: Adrenalectomy for metastatic disease to the adrenal glands. Br J Surg 83: 528-531, 1996.

39. Wade TP, Virgo KS, Li MJ, Callander PW, Longo WE and Johnson FE: Outcomes after detection of metastatic carcinoma of the colon and rectum in a national hospital system. J Am Coll Surg 182: 353-361, 1996. 